\title{
A controlled trial of the retraining of the sensory function of the hand in stroke patients
}

\author{
M Yekutiel, E Guttman
}

\begin{abstract}
A controlled trial of retraining of the sensory function of the hand was undertaken in hemiplegic patients after the period of spontaneous recovery. Twenty hemiplegic patients with sensory deficit in the hand, two or more years after stroke, received systematic retraining three times a week for six weeks. Sensation in the plegic hand was tested before and after this period in these patients and in 19 untreated control patients. The treated group showed large and significant gains on all sensory tests ( $P<0.001$ ), while no change occurred in the control group. It is concluded that somatosensory deficit can be alleviated even years after stroke and that rehabilitation for stroke patients should include sensory retraining for those with sensory deficit.
\end{abstract}

$(\Im$ Neurol Neurosurg Psychiatry 1993;56:241-244)

Functional evaluation of the stroke patient focuses on motor performance; motor deficit is seen as the primary reason for functional disability and therefore as the cardinal impairment to which physical and occupational therapy should be addressed. Sensory deficit has received less attention, although its adverse effect on functional outcome has been repeatedly documented..$^{1-5}$ Bobath, who developed one of the most widely used therapeutic approaches for adult hemiplegia, affirmed that "the patient with severe and persistent sensory deficit has a poor prognosis for functional recovery" " 6 but did not propose any steps for reducing this deficit. Bobath's and other therapies use sensory stimulation to facilitate movement, but few direct attempts have been made to promote sensory recovery after stroke, although there is some indirect evidence that this may be possible.

Potential for recovery and the critical role of retraining were demonstrated over fifty years $\mathrm{ago}^{7}$ in experiments on primates trained to discriminate manipulated objects by weight, texture and shape: this ability was lost after each of a series of parietal ablations but could be restored by retraining. Two reports advocated retraining of hand sensation in stroke patients, each report was supported by a single case study. ${ }^{89}$ Two controlled studies reported significant and lasting effects of training in sensory awareness: in response to double simultaneous stimulation $^{10}$ and in identification of the midline of the back in response to touch. ${ }^{11}$ While we found no published trial of re-education of hand sensation in hemiplegia, its value in peripheral nerve lesions was demonstrated by Wynn Parry ${ }^{12}$ both during and years after regeneration of sutured median nerve lesions. A large Russian study of war injuries of the upper extremity also showed in many detailed case reports the importance of active retraining for recovery of sensation in the hand.$^{13}$ It is clear from both these studies that recovery of sensation stemmed from the patient learning to make sense of unfamiliar somatic sensations through repeatedly matching them to vision and at the same time developing "tactics of perception"13 in the form of purposeful exploratory movements of the hand. This dual process is not spontaneous but requires intensive and directed practice: without this active retraining, the patient with a sensory deficient hand quickly learns to use it as little as possible.

In the absence of comparable data from patients with central lesions, these two studies of sensory recovery after peripheral lesions guided us in developing the method of sensory retraining used in this trial with stroke patients.

\section{Methods \\ a) Patients}

The study was authorised by the Helsinki Committee of Ben-Gurion University. Twenty patients aged over 40 years were recruited from the primary care clinics of the health insurance funds in the Beer-sheva area according to the following criteria: 1) two or more years after a major stroke with persisting sensory deficit in the hand; 2) no communication problem or significant cognitive or emotional disturbance; 3) informed consent. The ages of the patients ranged from 44-81 years (mean 64); there were 13 men and 7 women; 10 had had left hemisphere stroke (LHS) and 10 right (RHS), and time since stroke ranged from 2 to 18 years (mean $6 \cdot 2$ ). After the start of treatment an additional 20 patients were enrolled from the same source according to the same criteria to serve as controls for any possible learning effect of repeating the sensory examination. Non-availability of one subject at the second testing reduced their number to 19 . They were comparable to the treated group in having mean age 67 years, 9 having LHS, 10 RHS, after a mean period of $6 \cdot 2$ years. There were 8 men and 11 women. 


\section{b) Treatment}

Lessons of 45 minutes were given (by EG) to each patient in his home three times a week for six weeks. Sensory re-education was based on the following principles: 1) The nature and extent of sensory loss are explored together with the patient who is often unaware of it. 2) Emphasis is placed on sensory tasks which the patient can do, and these form the start and end of each lesson. 3) Sensory tasks for each patient are chosen which interest him or her and which promise to lead to sufficient failures and successes to promote learning. 4) Constant use is made not only of vision but also of the good hand to teach tactics of perception. 5) Frequent rests and change of subject are needed to maximise concentration. 6) No task or object used in the testing of sensation may be used in training.

Examples of subjects taught are: identification of the number of touches or lines and of numbers and letters drawn on the arm and hand; "find your (plegic) thumb" blindfolded; discrimination of shape, weight and texture of objects or materials placed in the hand; passive drawing. In the last subject, which was designed for these patients, the therapist holds the patient's hand, in which there is a pencil, and draws with it; the patient, unable to see their hand, tries to identify the figure drawn by choosing from four figures on a card which they can see, this being one of a series of cards of increasing difficulty. As proprioception improves, passive drawing leads to writing messages. The good hand and the plegic hand are used alternately.

\section{c) Tests of sensation}

All subjects were tested (by MY) before and after the six week period of treatment or non-treatment. A detailed protocol, including exact words to be used and duration of stimuli, was adhered to. No knowledge of results was given to the patient. There were four types of test:

1) Location of touch. The patient, unable to see his hand, was asked to identify the area touched with a blunt pencil by referring to a map of the (right or left) hand divided into 16 areas (14 phalanges, thenar and hypothenar). Twenty tests were made with constant pressure and duration.

2) Sense of elbow position. The plegic elbow of the blindfolded patient is flexed to a series of 10 angles, which he must match with his other arm.

Table 1 Percentage scores of 20 treated patients on sensory tests before and after treatment: mean (SE)

\begin{tabular}{|c|c|c|c|}
\hline Test & Before & After & $P$ \\
\hline $\begin{array}{l}1 \text { Location of touch } \\
2 \text { Sense of elbow position } \\
3 \text { Two-point discrimination } \\
4 \text { Stereognosis } \\
\text { Total score }\end{array}$ & $\begin{array}{l}33.7(5 \cdot 39) \\
77.2(2 \cdot 38) \\
25 \cdot 8(4 \cdot 61) \\
29 \cdot 5(4 \cdot 58) \\
41.7(2 \cdot 80)\end{array}$ & $\begin{array}{l}55 \cdot 1(5 \cdot 32) \\
85 \cdot 4(1 \cdot 48) \\
48 \cdot 1(3 \cdot 73) \\
56 \cdot 8(4 \cdot 85) \\
61 \cdot 7(3 \cdot 22)\end{array}$ & $\begin{array}{l}0.001 \\
0.001 \\
0.001 \\
0.0001 \\
0.0001\end{array}$ \\
\hline
\end{tabular}

ॠThese are two-tailed levels of statistical significance from $t$ tests on the difference between the means of paired samples.
3) Two-point discrimination (2-PD). This was tested on fingers, palm and forearm, using a wooden hexagon mounted with pins, with pairs of pin-heads separated by $1-5 \mathrm{cms}$. No attempt was made to establish threshold values for $2-\mathrm{PD}$, but the patient was asked to state if he felt one or two points; 32 locations were tested, 9 of these with one point.

4) Stereognosis (more precisely, tactile object recognition). A tray with 30 everyday objects was shown to the patient to find out what name or language they would use. The patient was blindfolded, and then asked to identify a fixed series of 20 of the objects (for example, a pill bottle, a duster, a doorhandle) placed in the hand. For those with poor motor function, the examiner moved the object in the patient's hand. Partial scores were given for varying levels of description without identification.

Correct answers on each test were expressed as percentage scores: the total score is the arithmetic mean of the four percentage scores. Statistical significance of differences between before and after mean scores was tested by two-tailed Student's $t$ test on the means of paired samples.

\section{Results}

The control group showed negligible changes in sensory scores between testing at outset and testing six weeks later. Pearson coefficients of correlation between the two sets of data give a test-retest reliability of $95 \%$ for the total score, while the individual tests 1-4 show reliability of $88 \%, 83 \%, 93 \%$ and $97 \%$ respectively. Change in the control group's mean scores on the four tests ranged from -1.0 to $+1 \cdot 2$, and the mean total score increased non-significantly from $43 \cdot 6$ to $44 \cdot 1$.

Scores for the treated patients (table 1) showed large gains with a high level of statistical significance $(P<0.001)$. The mean total score increased by 20 points from 41.7 to $61 \cdot 7$. The largest gain was for Stereognosis, the least for Sense of elbow position, but all reached the $0.1 \%$ level of significance. Results for treated and controls are shown in the figure.

This statistical analysis is based on the $t$ test for means of paired samples. While the patients' scores at the start approximated a normal distribution, the distribution of their gains after treatment is positively skewed to a degree which may reduce the reliability of the $t$ test, although this test has been described as "robust with respect to the non-normality of the population". ${ }^{14}$ We therefore took the precaution of a) normalising the raw data and recalculating $t$ values, and b) also using the Sign test. Both procedures tended to reduce the statistical significance of the differences between scores before and after treatment, but none fell below the $1 \%$ level.

The relation between individual gains on the four tests was examined by Pearson correlation. Gains in Stereognosis were significantly correlated with gains in both Location of touch $(r=+0.51)$ and 2-PD $(r=+0.61)$. 


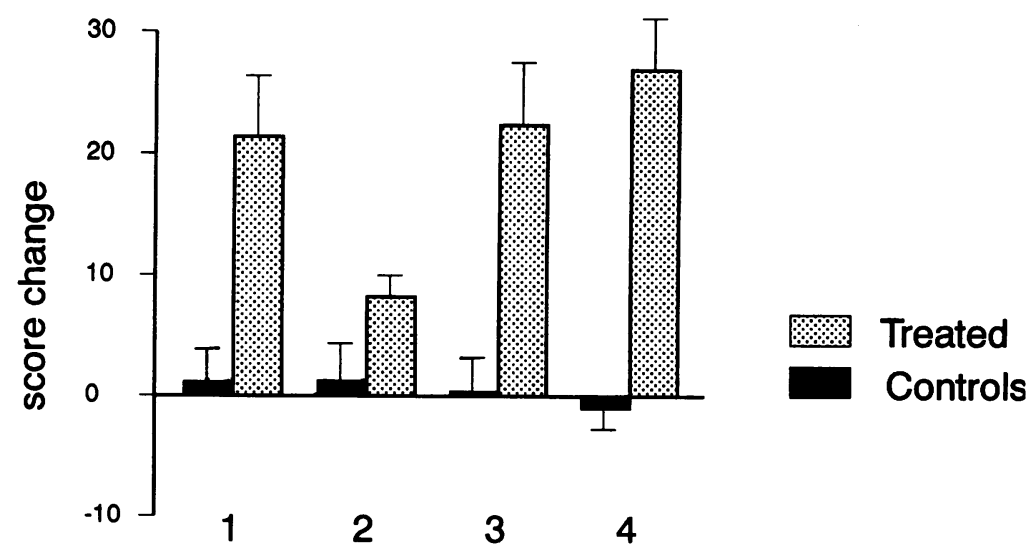

Figure Change in scores on tests 1-4: means and standard errors. (Maximum score on each test: 100) patients in this study confirms that improvement does not occur spontaneously after one year and that sensory deficit remains unchanged. It also rules out the possibility that the improved scores of the treated patients at the second testing could have resulted from their learning how to do the tests. Although allocation to control or treatment groups was not random, the untreated patients can be accepted as a legitimate control group in that the two groups came from the same source, met the same criteria and showed no differences approaching statistical significance in distribution by age, duration or laterality of stroke or in sensory status at outset.

The possibility of observer bias must be considered, since the examiner was in most cases not blind to the patient's allocation. Possible effects of this shortcoming were minimised by a) the use of a very precise protocol which allowed little variation in the way the tests were administered, and b) exact recording and objective scoring of patients' responses. It is therefore unlikely that the study findings were affected by bias.

The results indicate that the sensory function of the hand can be significantly improved in chronic hemiplegia by systematic retraining over a relatively short period. Physiotherapy tends to be given for long periods to stroke survivors. In the Manchester study, ${ }^{21} 42 \%$ of those treated by physiotherapy received it for over three months and $10 \%$ continued for more than a year. However, the value of this physiotherapy has been questioned. ${ }^{21} \mathrm{~A}$ number of critical studies have failed to demonstrate any therapeutic advantage for one approach over another, ${ }^{22-25}$ and a controlled trial demonstrating therapeutic effect-on either impairment or disability-has not yet been reported for any of the methods of physiotherapy commonly used for stroke patients. The moral and economic issues involved in treating this large patient population by unvalidated therapeutic methods call for a reconsideration of the nature of stroke and the development and evaluation of new approaches.

Central to current methods of physiotherapy for the stroke patient is the subject of muscle tone, and methods differ primarily in their use of inhibition and facilitation of tone. Since muscle tone is largely determined by spinal and lower brain centres, the techniques used to modify it emphasise "automatic" responses from the patient. The sensory stimuli used-such as passive or resisted movement, positioning, destabilising, brushing or rubbing with ice-undoubtedly influence muscle tone through these centres, but are likely to produce descending inhibition from higher sensory centres to which they represent "noise".

In addition to the primary motor deficit, mechanisms of sensory inhibition are involved to varying degrees in the stroke patient's non-use of the affected hand, whether this is due to central neglect or to learned non-use. Descending inhibition of 
input-both exteroceptive and proprioceptive-is unlikely to be combatted by the neuromuscular techniques listed above. But a wealth of little understood mechanisms of neuroplasticity ${ }^{26}$ may perhaps be exploited by therapy addressed to higher brain centres in the form of challenging and meaningful questions posed to the hand as sense organ. The intact peripheral nervous system can provide normal sensory input, but this is received in a reduced and distorted form whose interpretation has to be learned. These considerations underlie the method of sensory retraining developed and tested in this study. In contrast to the sensory stimuli currently used in physiotherapy, interesting and solvable tasks were used to encourage the brain/patient to seek and use input from the paretic hand. Modality-specific sensory tasks (described by two authorities as "academic" 1227 were avoided and emphasis placed on multi-modality problem-solving. The result was a large and highly significant improvement in the sensory function of the hand.

This result refers to the average performance of the treated group. Improvement in sensation, though not affected by patient's age or sex or duration of hemiplegia, varied greatly between patients, suggesting that the approach used was not in every case appropriate for the specific sensory disturbance. The relatively poor improvement in the ten patients with right hemisphere stroke (RHS) compared with the LHS patients suggests that the method used-although aimed at higher brain centres-may still be too "peripheral". On admission patients were screened for gross cognitive disturbance, but it is possible that the somatosensory deficit in some of the RHS patients was part of a wider perceptual disturbance or hemineglect which was neither explored nor addressed in this study. We believe that more sophisticated sensory-perceptual examination of patients, especially those with RHS, may enable sensory retraining to be better geared to the special problems of each patient.

The long-term aim of sensory retraining is to give the patient a more useful hand. However, no attempt was made either to evaluate or to promote use of the hand in daily life, though two patients reported finding that they could now use the hand more than before. Our impression was that intensive functional training would be needed to produce any change in the daily habits of these people who, years after major stroke, lived with assistance from relatives and social services, with little hope or motivation for change. Sensory retraining in the early rehabilitation of stroke patients should immediately harness any sensory recovery which may result in the improved use of the hand.

1 Van Buskirk C, Webster D. Prognostic value of sensory deficit in rehabilitation of hemiplegics. Neurology 1955:5:407-11.

2 Anderson AK. Sensory impairments in hemiplegia. Arch Phys Med Rehabil 1971:52:293-7.

3 Smith DL, Akhtar AJ, Garraway WM. Proprioception and spatial neglect after stroke. Age Ageing 1983:12:63-69.

4 Zeman BD, Yiannikas C. Functional prognosis in stroke use of somato-sensory evoked potentials. $\mathcal{f}$ Neuro Neurosurg Psychiatry 1989:52:242-7.

5 Reding MJ. A model stroke classification scheme and its use in outcome research. Stroke 1990:21 (suppl II):II-35II-37.

6 Bobath B. Adult hemiplegia: evaluation and treatment. London: Heinemann, 1970:25.

7 Ruch TC, Fulton JF, German WJ. Sensory discrimination in monkey, chimpanzee and man after lesions of the in monkey, chimpanzee and man after lesions of

parietal lobe. Arch Neurol Psychiatry 1938:39:919-38.
8 Forster FM, Shields CD. Cortical sensory defects causing disability. Arch Phys Med Rehabil 1959:40:56-61.

9 Dannenbaum RM, Dykes RW. Sensory loss in the hand after sensory stroke: therapeutic rationale. Arch Phys Med Rehabil 1988:69:833-9.

10 Goldman H. Improvement of double simultaneous stimulation perception in hemiplegic patients. Arch Phys Med Rehabil 1966:47:681-7.

11 Weinberg J, Diller L, Gordon WA et al. Training sensory awareness in people with right brain damage. Arch Phys Med Rehabil 1979:60:491-6.

12 Wynn Parry CB, Salter M. Sensory reeducation after median nerve lesions. The Hand 1975:8:250-7.

13 Leont'ev AN, Zaporozhets AV. Rehabilitation of hand function. Oxford: Pergamon, 1960:39-60.

14 Lapin L Statistics: meaning and method. New York: Harcourt, 1975:318.

15 Prescott RJ, Garraway WM, Akhtar AJ. Predicting functional outcome following acute stroke using a standard clinical examination. Stroke 1982:13:641-7.

16 Wade DT, Hewer RL, Wood VA, Skilbeck CE, Ismail HM. The hemiplegic arm after stroke: measuremen and recovery. $\mathcal{F}$ Neurol Neurosurg Psychiatry 1983:46: $521-4$.

17 Olsen TS. Arm and leg paresis as outcome predictors in stroke rehabilitation. Stroke 1990:21:247-51.

18 Kelly-Hayes $M$. Time intervals, survival, and destination: three crucial variables in stroke outcome research. Stroke 1990:21 (suppl II):II-24-II-26.

19 Lehmann JF, DeLateur BJ, Fowler RS, et al. Stroke: does rehabilitation affect outcome? Arch Phys Med Rehabil 1975:56:375-82.

20 Tangeman PT, Banaitis DA, Williams AK. Rehabilitation of chronic stroke patients: changes in functional perfor mance. Arch Phys Med Rehabil 1990:71:876-80.

21 Brocklehurst JC, Andrews K, Richards B, Laycock PJ. How much physiotherapy for patients with stroke? BMf 1978:1:1307-10.

22 Logigian MK, Samuels MA, Falconer J. Clinical exercise trial for stroke patients. Arch Phys Med Rehabil 1983:64:364-7.

23 Dickstein R, Hocherman S, Pillar T, Shaham R. Stroke rehabilitation: three exercise therapy approaches. Phys Ther 1986:66:1233-8.

24 Lord JP, Hall K. Neuromuscular reeducation versus traditional programs for stroke rehabilitation. Arch Phys Med tional programs for stro

25 Wagenaar RC, Meijer OG, Van Wieringen PCW, et al. Wagenaar RC, Meijer OG, Van Wieringen PCW, et al.
The functional recovery of stroke: a comparison between neuro-developmental treatment and the Brunnstrom method. Scan F Rehabil Med 1990:22:1-8.

26 Bach-y-Rita P. Brain plasticity as a basis of the development of rehabilitation procedures for hemiplegia. Scand f Rehabil Med 1981:13:73-83.

27 Moberg E. Criticism and study of methods for examining sensibility in the hand. Neurology 1962:12:8-19. 